\title{
Obesidad, morfología corporal y presión arterial en grupos urbanos y rurales de Yucatán
}

Pedro Arroyo, MC, MSP, MSc, (I) Victoria Fernández, LEO,(') Alvar Loría, PhD, (2) Jeannette Pardío, LN, ${ }^{(1)}$ Hugo Laviada, MC, (3) Lizardo Vargas-Ancona, MC, ${ }^{(3)}$ Ryk Ward, ${ }^{\dagger}$ PhD. ${ }^{(4)}$

Arroyo P, Fernández V, Loría A, Pardío J, Laviada H, Vargas-Ancona L, Ward R. Obesidad, morfología corporal y presión arterial en grupos urbanos y rurales de Yucatán. Salud Publica Mex 2007;49:274-285.

\section{Resumen}

Objetivo. Caracterizar la antropometría y presión arterial de adultos del estado de Yucatán, México. Material y métodos. Se evaluaron diferencias rural-urbanas por grupos sexo-edad en peso, talla, circunferencias de cintura y cadera, y presión arterial en 3/3 adultos de origen urbano y 27| del rural, de Yucatán. Resultados. No hubo diferencias rural-urbanas en prevalencias de obesidad y sobrepeso, $y$ en hipertensión los urbanos tuvieron valores marginalmente mayores. Se encontró mayor prevalencia rural de cintura anormal sólo en mujeres y hombres jóvenes. La comparación con dos encuestas nacionales y una regional (mixtecos rurales) mostró obesidad similar a la notificada en la Encuesta Nacional de Salud 2000 (ENSA) y mayor que mixtecos y la informada en la Encuesta Nacional de Enfermedades Crónicas 1993 (ENEC). La prevalencia de cintura anormal fue intermedia entre la indicada en la Encuesta Nacional de Salud y Nutrición 2006 (ENSANUT) y mixtecos, y la de hipertensión intermedia entre la notificada en la ENEC y mixtecos. Conclusiones. Las poblaciones maya y mestiza de Yucatán presentaron alta prevalencia de obesidad y cintura anormal que no se acompañaron de prevalencia mayor de hipertensión. Esta observación requiere confirmación.

Palabras clave: sobrepeso; obesidad; hipertensión; prevalencia; población;Yucatán; México
Arroyo P, Fernández V, Loría A, Pardío J, Laviada $\mathrm{H}$, Vargas-Ancona L, Ward R.

Obesity, body morphology, and blood

pressure in urban and rural population groups of Yucatan. Salud Publica Mex 2007;49:274-285.

\section{Abstract}

Objective. To characterize body morphology and blood pressure of adults of the Mexican state of Yucatan. Material and Methods. Rural-urban differences in weight, height, waist, and hip circumferences, and blood pressure were analyzed in 313 urban and 27I rural subjects. Results. No rural-urban differences in prevalence of obesity and overweight were found. Hypertension was marginally higher in urban subjects. Rural abnormal waist circumference was higher in young men and young women. Comparison with two national surveys and a survey in the aboriginal population (rural mixtecos) showed similar prevalence of obesity as ENSA-2000 and higher than mixtecos and ENEC-1993. Abnormal waist circumference was intermediate between ENSANUT-2006 and mixtecos and hypertension was intermediate between ENEC and mixtecos. Conclusion. The Maya and mestizo population of Yucatan showed a high prevalence of obesity and abnormal waist circumference not accompanied by a comparable higher hypertension frequency. This finding requires further confirmation.

Key words: overweight; obesity; hypertension; prevalence; population;Yucatán; Mexico

(I) Fondo Nestlé para la Nutrición, Fundación Mexicana para la Salud. México

(2) Instituto Nacional de Ciencias Médicas y Nutrición Salvador Zubirán. México

(3) Hospital O'Horán, Facultad de Medicina de la Universidad Autónoma de Yucatán. México

(4) Institute of Biological Anthropology, Oxford University. EUA

Fecha de recibido: 31 de octubre de 2005 - Fecha de aprobado: 20 de abril de 2007

Solicitud de sobretiros: MSc Pedro Arroyo. Fundación Mexicana para la Salud.

Periférico Sur 4809. 14610 Tlalpan, DF, México.

Correo electrónico: parroyo@fondonestlenutricion.org.mx 
$\mathrm{L}$ a obesidad es un factor de riesgo cardiovascular Jasociada a comorbilidades como la hipertensión arterial, la diabetes mellitus tipo 2 y las dislipidemias, entre otras. ${ }^{1-3}$ México comparte este problema de salud con otras naciones. Para explicarlo, se han invocado fenómenos macroeconómicos como la urbanización y la consiguiente reducción de la actividad física, así como el aumento de la disponibilidad de alimentos baratos con densidad energética alta. ${ }^{4}$ Sin embargo, las diferencias regionales y las observadas según estrato socioeconómico al interior de países como México no son fáciles de explicar desde esa perspectiva. Una alternativa para avanzar en el conocimiento de estos factores consiste en estudiar las diferencias regionales de prevalencia en función de los cambios recientes del estilo de vida de la población mexicana. Esos cambios han tenido una dinámica variable según las diferencias geográficas, étnicas, de desarrollo industrial y urbanístico, y han modificado la cultura alimentaria y los hábitos de actividad física de las poblaciones.

En este trabajo se comparan las prevalencias de obesidad y de hipertensión en poblaciones de origen urbano y rural en el estado de Yucatán, con las de encuestas nacionales y con las de otros grupos de población mexicana. Este estudio forma parte de una serie con otros dos trabajos donde los autores han presentado las prevalencias de diabetes y dislipidemias, así como descripciones de los patrones de alimentación y de actividad física. ${ }^{*}$ La hipótesis que subyace a estos trabajos es que las diferencias de prevalencias de obesidad e hipertensión de las poblaciones rural y urbana entre sí, y con respecto de la población del país, están relacionadas con estilos de vida rural/urbana específicos de Yucatán. Se considera que estos trabajos, pese a ser principalmente descriptivos, pueden contribuir a sentar algunas bases que permitan entender mejor las diferencias regionales de la epidemiología de las enfermedades crónicas relacionadas con la nutrición.

\section{Material y métodos}

\section{Encuesta urbana}

El estudio de la población de Mérida se llevó a cabo de julio a diciembre de 1997. La selección de la muestra

\footnotetext{
* Loría A, Laviada H, Medina-Escobedo M, Villanueva-Jorge S, Valles V, Fernández V, et al. Prevalencias de diabetes y dislipidemias en grupos urbanos y rurales de Yucatán. Sometido a Salud Publica Mex, 2007.

Pardío J, Loría A, Fernández V, Arroyo P. Patrones de alimentación y de actividad física en grupos urbanos y rurales de Yucatán. Sometido a Salud Publica Mex, 2007.
}

poblacional se hizo mediante un muestreo probabilístico polietápico. La distribución por edad y sexo se calculó con base en el censo de población de $1990^{5}$ y el tamaño de la muestra se diseñó para que fuera representativa de los sujetos de 2 a 75 años de edad de ambos sexos, de los estratos socioeconómicos medio-bajo y bajo (excluyendo los sectores de asentamientos irregulares). Esta muestra representa a 646740 sujetos de la población total de Mérida (311 079 hombres y 335661 mujeres;213 442 de estrato medio-bajo y 433298 del bajo). La estratificación socioeconómica se hizo según los criterios de la Asociación Mexicana de Agencias de Investigación de Mercados y Opinión Pública, ${ }^{6}$ que reúne a las 19 agencias que realizan $80 \%$ de la investigación de mercados en México. Los estratos se definieron por la apariencia de las casas, la disponibilidad de servicios, las vías de comunicación y los medios de transportación, entre otras características. En las zonas identificadas se seleccionaron manzanas en forma aleatoria y se visitaron todas las viviendas de las manzanas seleccionadas. Se realizó una visita para hacer la invitación a las personas seleccionadas, proporcionando información sobre los procedimientos a realizar. En cada vivienda se invitó a participar a un miembro de la familia, hasta completar la cuota establecida para cada grupo de edad, sexo y estrato. Los resultados aquí presentados se refieren a 313 sujetos de 20 a 75 años de edad (185 mujeres y 128 hombres).

\section{Encuesta rural}

La muestra rural se obtuvo de dos comunidades mayas de menos de 1200 habitantes cuyos nombres son Ucí, perteneciente al municipio de Motul, y San Rafael, del municipio de Maxcanú. El estudio se llevó a cabo entre agosto y septiembre de 2000, e incluyó a la tercera parte de la población de 20 a 75 años de ambas comunidades. Se seleccionaron las familias que tenían al padre y a la madre biológicos, al menos un hijo mayor de 15 años, y que vivieran en el mismo hogar. Además, se incluyó a un hermano del padre o la madre, residente en la comunidad. En ambas comunidades el trabajo de campo se organizó con el apoyo de Asistentes Rurales del Programa IMSS-Oportunidades. Se explicó a cada familia acerca de los objetivos y procedimientos del estudio, con el auxilio de personal bilingüe. Las familias aceptantes fueron citadas en locales adaptados en cada comunidad, donde enfermeras capacitadas y estandarizadas realizaron los procedimientos. Los resultados que se muestran para ambas comunidades corresponden a los participantes de 20 a 75 años de edad que recibieron una evaluación completa. En este análisis se incluyó a 271 sujetos de 20 a 75 años (157 mujeres y 114 hombres). Los datos de 
ambas comunidades se presentan englobados, ya que sus habitantes comparten características semejantes como el ser bilingües, estar dedicados a actividades agrícolas y de maquila, y migrar a la ciudad de Mérida por cuestiones laborales.

Los estudios rural y urbano se realizaron de acuerdo con los lineamientos éticos vigentes en las fechas en que se implementaron y fueron avalados por el Comité de Ética del Hospital O’Horán de Mérida. En el caso del estudio urbano, se obtuvo el consentimiento informado por escrito de cada sujeto o de la persona responsable de su cuidado. En el caso del estudio rural se obtuvo el consentimiento de manera verbal, en presencia de personal del Programa IMSS-Oportunidades como testigo y por escrito por parte de un representante comunitario.

\section{Antropometría}

La talla se midió con una cinta métrica adherida a un estadímetro portátil de madera, construido para este fin. El peso se cuantificó con una báscula electrónica digital marca Tanita, modelo BWB-600, con sensibilidad de $100 \mathrm{~g}$ y variabilidad cercana a cero. Dos observadores independientes midieron las circunferencias de cintura y de cadera con una cinta métrica de fibra de vidrio, y la unidad de análisis fue el promedio de las mediciones. Las enfermeras del estudio urbano fueron capacitadas y certificadas por el Instituto de Investigaciones Antropológicas de la Universidad Nacional Autónoma de México (UNAM) para llevar a cabo las mediciones antropométricas. La capacitación se terminó hasta que las enfermeras no discreparon significativamente del experto. En el estudio rural hubo ocho enfermeras capacitadas y estandarizadas por uno de los autores de este trabajo (VF). Se continuó con la capacitación hasta que las enfermeras estuvieron dentro de $\pm 2 \mathrm{~mm}$ de talla y \pm 5 de cintura en relación con la experta. Las cuatro mediciones antropométricas permitieron calcular el índice de masa corporal (IMC) mediante la ecuación IMC = peso en $\mathrm{kg}$ / cuadrado de talla en $\mathrm{m}$ (unidad $=\mathrm{kg} / \mathrm{m}^{2}$ ) y el índice cintura/ cadera (ICC) dividiendo la CCIN entre la CCAD (unidad = tanto por uno ya que se divide $\mathrm{cm} / \mathrm{cm}$ ). Con el IMC se calcularon las prevalencias de sobrepeso $\left(\mathrm{IMC}=25\right.$ a $29.9 \mathrm{~kg} / \mathrm{m}^{2}$ ) y obesidad $(\mathrm{IMC}>=$ $30 \mathrm{~kg} / \mathrm{m}^{2}$ ). Con la cintura se estableció el porcentaje de sujetos con valores alterados de acuerdo a dos puntos de corte establecidos por la Organización Mundial de la Salud (OMS). ${ }^{7,8}$ Estos puntos de corte son diferentes para mujeres y hombres e identifican niveles medio y alto de riesgo.

\section{Presión arterial}

En la encuesta urbana, la medición de la presión arterial corrió a cargo de nueve enfermeras, quienes conformaron tres equipos de tres miembros y fueron capacitadas y estandarizadas técnicamente por un experto del Instituto Nacional de Cardiología Ignacio Chávez de México, hasta que los equipos alcanzaron una buena concordancia interobservadores dentro y entre equipos. En la encuesta rural, las enfermeras fueron capacitadas y estandarizadas por uno de los autores de este trabajo (VF) hasta lograr que las lecturas digitales del Omrom fuera idénticas a las de la experta. En el estudio de la muestra urbana se utilizaron tres esfigmomanómetros de los llamados "random-zero" marca Hawksley \& Son, que permiten tres lecturas (sístólica, diastólica y una lectura cero) y a las presiones diastólica y sistólica se les resta la lectura cero para "corregirlas". Dos observadores realizaron las mediciones en el hogar, registrando las presiones sistólica (PAS) y diastólica (PAD) según fases I y V de Korotkoff, respectivamente; más tarde, se promediaron los valores de los dos observadores. En la encuesta rural, las mediciones se hicieron en locales adaptados para esos fines, utilizando tres esfigmomanómetros electrónicos de tipo oscilométrico digital (modelo HEM-412C de Omron). Se colocó a la persona en reposo y sentada, y se registró una primera medición preliminar y tres subsecuentes con cinco minutos de intervalo entre mediciones, siguiendo las recomendaciones del VI informe del Joint National Committee for the Prevention, Detection, Evaluation and Treatment of High Blood Pressure de los Institutos Nacionales de Salud de Estados Unidos de América (JNC VI). ${ }^{9}$ Los datos de análisis fueron el promedio de las tres últimas mediciones. La hipertensión arterial se definió según los criterios del JNC VI, ${ }^{9}$ de modo que los sujetos con PAS > $=140 \mathrm{mmHg}$ y/o PAD $>=90 \mathrm{mmHg}$ fueron considerados hipertensos.

Se sabe ahora que los métodos utilizados en este estudio para medir la presión de urbanos y rurales ("random zero" en urbanos y Omron oscilométrico en rurales) no son comparables. Por ello, se llevó a cabo un estudio posterior donde se compararon los esfigmomanómetros del estudio. ${ }^{10}$ Un solo observador realizó la comparación en un grupo de 33 sujetos asistentes a un servicio de detección y control de hipertensión arterial. Se compararon los tres aparatos RZ (random zero) del estudio urbano y los cuatro Omron del rural. A cada sujeto se le hicieron mediciones duplicadas con dos o más de los aparatos RZ y Omron y se alternó el orden en 
que fueron usados los dos tipos de esfigmomanómetro en los 33 sujetos. Los factores que se aplicaron a las presiones sistólica y diastólica de "random zero" fueron el promedio de las discrepancias entre las lecturas Omron que resultaron sistemáticamente más altas que las de los aparatos "random zero" en todo el rango de PAS y PAD estudiado. Los factores de corrección fueron similares en el rango PAS = 90 a $140 \mathrm{mmHg}$ y $\mathrm{PAD}=70$ a $110 \mathrm{mmHg}$. En pocas instancias, se observó que el factor tendía a bajar en sujetos con valores inferiores a estos límites y que, por el contrario, tendía a subir ligeramente en los valores superiores a dichos límites. Sin embargo, se optó por usar factores únicos de 1.04 y 1.07, ya que los PAS y PAD fuera de estos límites corresponden a sujetos francamente normotensos o claramente hipertensos. Así entonces, las correcciones efectuadas no cambiaron la clasificación de los individuos estudiados con el aparato "random zero"cuando éstos estuvieron fuera de los límites antes mencionados. En consecuencia, los datos de PAS y PAD medidos con RZ multiplicados por 1.04 y 1.07 respectivamente, son los que se presentan en este estudio. Se optó por transformar los datos RZ a Omron porque los estudios que se llevan a cabo actualmente se realizan con esfigmomanómetros Omron.

\section{Análisis estadístico}

Las diferencias rural-urbano se evaluaron mediante las pruebas de ji cuadrada y de diferencia de medianas de Mann-Whitney. Esta última se utilizó en lugar de la prueba de Student por la alta dispersión de algunas variables que tuvieron coeficientes de variación superior a 50\%. En las comparaciones con otras series se recurrió a la prueba de ji cuadrada para series pequeñas, así como a la diferencia de proporciones de una sola muestra para las comparaciones con las series de varios miles de sujetos. ${ }^{11}$

\section{Resultados}

\section{Diferencias rural-urbanas al interior de los grupos}

Los resultados se muestran en ocho grupos conformados de acuerdo con el sexo (mujer y hombre), la edad (20-49 y $50+$ años) y el origen (rural y urbano). Estos grupos se designan en los cuadros y texto con tres letras mayúsculas correspondientes a sexo $(\mathrm{M}=$ mujer $/ \mathrm{H}=$ hombre $)$, edad ( $\mathrm{J}=$ jóvenes $/ \mathrm{M}=$ maduros) y origen $(\mathrm{R}=\mathrm{rural} / \mathrm{U}=$ urbano). Así, la sigla MJR corresponde a mujeres jóvenes rurales; la de MJU a mujeres jóvenes urbanas, y HJR a hombres jóvenes rurales, etc. Las diferencias rural-urbano siempre se hicieron al interior de los grupos (MJR vs. MJU, MMR vs. MMU, HJR vs. HJU y HMR vs. HMU), esto es, comparando grupos iguales en sexo y edad pero diferentes en origen.

En el cuadro I se muestra el análisis estadístico de las diferencias Rur-Urb de seis variables antropométricas y de las presiones sistólica y diastólica. Se observa que los promedios del índice de masa corporal (IMC) de los ocho grupos de sujetos oscilaron entre 25.8 y $30.7 \mathrm{~kg} / \mathrm{m}^{2}$. Así entonces, en los ocho grupos la media sobrepasó el valor de corte que identifica a los sujetos con sobrepeso (IMC >=25) y hubo un grupo (mujeres maduras rurales) cuya media sobrepasó el límite de obesidad (IMC>=30). La variabilidad fue sensiblemente igual en los grupos rurales y urbanos, v.gr. los coeficientes de variación (CV) oscilaron entre 12 y $18 \%$. La talla fue, en general, baja y con muy poca variabilidad intragrupal (CVs entre $3 \mathrm{y}$ $6 \%)$; los promedios de peso corporal estuvieron, por el contrario, por arriba de $60 \mathrm{~kg}$ y mostraron una variabilidad similar a la de IMC (CVs de 13 a 20\%).

El análisis inferencial del cuadro I muestra que las y los jóvenes rurales tuvieron un IMC significativamente mayor y una talla significativamente menor que sus contrapartes urbanas. Por otra parte, los datos de IMC $\mathrm{y}$ talla fueron muy parecidos en los grupos maduros rurales y urbanos.

El índice cintura/ cadera promedio estuvo cercano al nivel de riesgo alto de este índice (ICC $=>1$ ) en los cuatro grupos rurales (medias de 0.93 a 0.98 ), en tanto que fue menor en los grupos urbanos (0.81 a 0.93 ). La variabilidad, por otro lado, fue similar en rurales y urbanos (CVs de 4 a 9\%). Las diferencias de medias alcanzaron significancia, de modo que los rurales tuvieron un ICC significativamente mayor que los urbanos. En el mismo cuadro puede verse que esta diferencia se debió, sobre todo, a una cintura significativamente mayor en los grupos rurales, aunque también participó una tendencia a una mayor cadera de los urbanos. De esta manera, el mayor ICC rural obedeció a una mayor cintura abdominal y a una menor cadera.

En el cuadro I también se presentan los datos de las presiones arteriales sistólica (PAS) y diastólica (PAD) en los ocho grupos de sujetos. Las medias de PAS fueron menores en los rurales (104 a $124 \mathrm{mmHg}$ ) que en los urbanos (117 a $138 \mathrm{mmHg}$ ). En contraste, las medias de PAD fueron similares en rurales (70 a $75 \mathrm{mmHg}$ ) y urbanos (67 a $74 \mathrm{mmHg}$ ) de modo que, prácticamente, no hubo diferencias intergrupos en PAD. Llama la atención la mayor variabilidad en el PAD urbano (CVs de 19 a $24 \%$ ) que en el PAD rural (12 a 14\%), lo cual no ocurrió en la variabilidad del PAS urbano.

\section{Prevalencia de anormalidades}

En el cuadro II se muestran las prevalencias de obesidad y sobrepeso, las de valores anormales de ICC y de 
Cuadro I

Diferencias RURAL-URbana de datos ANTROPOMÉtricos y PRESIÓn ARTERIAL EN OCHO GRUPOS DE GÉNERO-EDAD

Grupos rurales

Grupos urbanos

Diferencias rural / urbana

\begin{tabular}{|c|c|c|c|c|c|c|c|c|c|c|c|c|c|c|}
\hline Variable & Grupo & $N$ & Media & $D E$ & $C V$ & Grupo & $N$ & Media & $D E$ & $C V$ & $U$ & $P$ & & \\
\hline IMC & MJR & 119 & 29.3 & 5.0 & 17 & MJU & 96 & 27.8 & 4.8 & 17 & 2.0 & 0.04 & & $\mathrm{R}>\mathrm{U}$ \\
\hline \multirow[t]{3}{*}{$\left(\mathrm{kg} / \mathrm{m}^{2}\right)$} & MMR & 38 & 29.8 & 5.3 & 18 & MMU & 89 & 30.7 & 5.0 & 16 & -0.8 & 0.44 & & \\
\hline & $\mathrm{HJR}$ & 63 & 28.1 & 3.5 & 12 & $\mathrm{HJU}$ & 58 & 25.8 & 4.1 & 16 & 3.1 & 0.00 & & $R>U$ \\
\hline & HMR & 51 & 27.9 & 3.6 & 13 & $\mathrm{HMU}$ & 70 & 26.8 & 3.9 & 15 & 1.4 & 0.15 & & \\
\hline Talla & MJR & 119 & 147 & 4.6 & 3 & MJU & 96 & 149 & 6.3 & 4 & -2.1 & 0.03 & & $U>R$ \\
\hline \multirow[t]{3}{*}{$(\mathrm{cm})$} & MMR & 38 & 144 & 5.4 & 4 & MMU & 89 & 146 & 6.2 & 4 & -1.6 & 0.11 & & \\
\hline & $\overline{H J R}$ & 63 & 159 & 4.4 & 3 & HJU & 58 & 163 & 9.3 & 6 & -3.1 & 0.00 & & $U>R$ \\
\hline & HMR & 51 & 158 & 5.8 & 4 & HMU & 70 & 158 & 7.2 & 5 & 0.4 & 0.70 & & \\
\hline Peso & MJR & 119 & 63.5 & 11.3 & 18 & MJU & 96 & 61.9 & 11.0 & 18 & 1.0 & 0.30 & & \\
\hline \multirow[t]{3}{*}{$(\mathrm{kg})$} & MMR & 38 & 62.3 & 12.2 & 20 & MMU & 89 & 65.7 & 12.8 & 19 & -1.1 & 0.25 & & \\
\hline & $\mathrm{HJR}$ & 63 & 70.9 & 8.9 & 13 & HJU & 58 & 68.9 & 12.5 & 18 & 1.8 & 0.08 & $M$ & $\mathrm{R}>\mathrm{U}$ \\
\hline & $\overline{H M R}$ & 51 & 69.5 & 10.7 & 15 & HMU & 70 & 67.2 & 12.3 & 18 & 1.1 & 0.28 & & \\
\hline \multirow{4}{*}{$\begin{array}{l}\text { Cintura } \\
(\mathrm{cm})\end{array}$} & $M J R$ & 119 & 94.2 & 13.7 & 15 & MJU & 96 & 81.9 & 10.4 & 13 & 6.7 & 0 & & $\mathrm{R}>\mathrm{U}$ \\
\hline & MMR & 38 & 98.8 & 13.1 & 13 & MMU & 89 & 91.7 & 10.3 & 11 & 2.7 & 0.01 & & $\mathrm{R}>\mathrm{U}$ \\
\hline & $\mathrm{HJR}$ & 63 & 92.3 & 8.5 & 9 & $\mathrm{HJU}$ & 58 & 86.1 & 10.8 & 13 & 3.6 & 0 & & $R>U$ \\
\hline & $\overline{\mathrm{HMR}}$ & 50 & 95.3 & 9.1 & 10 & HMU & 70 & 91.7 & 9.9 & 11 & 2.1 & 0.04 & & $R>U$ \\
\hline \multirow{4}{*}{$\begin{array}{l}\text { Cadera } \\
(\mathrm{cm})\end{array}$} & MJR & 119 & 101 & 9.7 & 10 & MJU & 96 & 101 & 9.2 & 9 & -0.6 & 0.58 & & \\
\hline & MMR & 38 & 100 & 11.3 & 11 & MMU & 89 & 106 & 10.9 & 10 & -2.1 & 0.04 & & $U>R$ \\
\hline & $\mathrm{HJR}$ & 62 & 97 & 5.9 & 6 & HJU & 58 & 97 & 8.1 & 8 & -0.2 & 0.87 & & \\
\hline & HMR & 50 & 97 & 7.2 & 7 & $\mathrm{HMU}$ & 70 & 98 & 7.8 & 8 & -0.8 & 0.41 & & \\
\hline \multirow{4}{*}{$\begin{array}{l}\text { ICC } \\
\text { (razón) }\end{array}$} & MJR & 119 & 0.93 & 0.08 & 9 & MJU & 96 & 0.81 & 0.07 & 8 & 9.5 & 0 & & $\mathrm{R}>\mathrm{U}$ \\
\hline & MMR & 38 & 0.98 & 0.06 & 6 & MMU & 89 & 0.87 & 0.06 & 7 & 7.1 & 0 & & $\mathrm{R}>\mathrm{U}$ \\
\hline & $\mathrm{HJR}$ & 62 & 0.95 & 0.05 & 5 & HJU & 58 & 0.89 & 0.07 & 8 & 5.5 & 0 & & $\mathrm{R}>\mathrm{U}$ \\
\hline & HMR & 50 & 0.98 & 0.04 & 4 & HMU & 70 & 0.93 & 0.06 & 6 & 4.9 & 0 & & $R>U$ \\
\hline \multirow{4}{*}{$\begin{array}{l}\text { PAS } \\
(\mathrm{mmHg})\end{array}$} & MJR & 119 & 104 & 14,7 & 14 & MJU & 96 & 117 & 15.2 & 13 & -6.3 & 0 & & $U>R$ \\
\hline & MMR & 38 & 121 & 18,2 & 15 & MMU & 89 & 138 & 19.3 & 14 & -4.5 & 0 & & $U>R$ \\
\hline & $\mathrm{HJR}$ & 63 & 113 & 12,1 & 11 & HJU & 58 & 121 & 12.3 & 10 & -3.4 & 0.00 & & $U>R$ \\
\hline & HMR & 51 & 124 & 22,7 & 18 & HMU & 70 & 133 & 21.7 & 16 & -3.0 & 0.00 & & $U>R$ \\
\hline \multirow{4}{*}{$\begin{array}{l}\text { PAD } \\
(\mathrm{mmHg})\end{array}$} & $M J R$ & 119 & 70,1 & 9,3 & 13 & MJU & 96 & 67.1 & 16.3 & 24 & 1.8 & 0.07 & M & $\mathrm{R}>\mathrm{U}$ \\
\hline & MMR & 38 & 72,6 & 9,1 & 13 & MMU & 89 & 73.7 & 15.2 & 21 & -0.5 & 0.62 & & \\
\hline & $\mathrm{HJR}$ & 63 & 74,5 & 8,7 & 12 & HJU & 58 & 73.9 & 14.2 & 19 & 0.4 & 0.66 & & \\
\hline & HMR & 51 & 75,4 & 10,3 & 14 & HMU & 70 & 74.3 & 16.3 & 22 & -0.2 & 0.83 & & \\
\hline
\end{tabular}

IMC = índice de masa corporal

ICC = índice cintura/cadera

PAS y PAD = presión arterial sistólica y diastólica

$U=$ valor de prueba $U$ de Mann-Whitney

$\mathrm{P}=$ probabilidad NO diferencia rural-urbana al interior de los grupos

Se añade una $M$ si la $p$ es marginal $(p=0.05$ a 0.10$)$

MJR y MJU = mujeres jóvenes rurales y urbanas

MMR y MMU = mujeres maduras rurales $y$ urbanas

HJR y HJU = hombres jóvenes rurales y urbanos

HMR y HMU = hombres maduros rurales $y$ urbanos 
cintura, y las de presiones arteriales sistólica y diastólica anormales. En las variables antropométricas se presentan dos niveles de riesgo (alto y medio) y, para facilitar la presentación, se hará referencia a los niveles con una letra final A (si es de riesgo alto) o de una $\mathrm{M}$ (riesgo medio). Así por ejemplo, los sujetos obesos estarán en el grupo IMC-A (IMC de riesgo alto) y los de sobrepeso en IMC-M (IMC de riesgo medio). Los puntos de corte para establecer los niveles de riesgo se anotan en el propio cuadro II.
En el mismo cuadro se aprecia que las prevalencias de sujetos con obesidad y sobrepeso fueron altas tanto en aquellos de origen rural como en los urbanos. La suma de obesidad y sobrepeso arrojó prevalencias altas que oscilaron entre 55 y $90 \%$ en los ocho grupos. Por otra parte, se observa que en lo concerniente a sobrepeso y obesidad prácticamente no hubo diferencias significativas entre los grupos rurales y urbanos. Hubo, asimismo, un grupo que no siguió la tendencia general Rur $>$ Urb de los demás: las mujeres maduras fueron las únicas

\section{Cuadro II}

Prevalencia de obesidad, sobrepeso e hipertensión y diferencias RURAL-URbanas en 8 grupos de SeXo-Género-edad Grupos RURALES

Grupos URBANOS

DIFERENCIAS rural / urbana

\begin{tabular}{|c|c|c|c|c|c|c|c|c|c|c|c|c|}
\hline Anormalidad & Grupo & $N I$ & N2 & Prevalencia & Grupo & $N I$ & N2 & Prevalencia & Chi & $P$ & & \\
\hline IMC-A & $M J R$ & 119 & 49 & $41 \%$ & MJU & 96 & 34 & $35 \%$ & 0.7 & 0.39 & & \\
\hline $\mathrm{IMC} \geq 30 \mathrm{~kg} / \mathrm{m} 2$ & MMR & 38 & 17 & $45 \%$ & MMU & 89 & 43 & $48 \%$ & 0.1 & $0.7 \mathrm{I}$ & & \\
\hline \multirow{2}{*}{ (Obesidad) } & $\mathrm{HJR}$ & 63 & 14 & $22 \%$ & $\mathrm{HJU}$ & 58 & 9 & $16 \%$ & 0.9 & 0.35 & & \\
\hline & HMR & 51 & 16 & $31 \%$ & $\mathrm{HMU}$ & 70 & 19 & $27 \%$ & 0.3 & 0.61 & & \\
\hline IMC-M & MJR & 119 & 45 & $38 \%$ & MJU & 96 & 34 & $35 \%$ & 0.1 & 0.72 & & \\
\hline $\mathrm{IMC}=25$ a 29.9 & MMR & 38 & 14 & $37 \%$ & MMU & 89 & 37 & $42 \%$ & 0.2 & 0.62 & & \\
\hline \multirow[t]{2}{*}{ (Sobrepeso) } & $\mathrm{HJR}$ & 63 & 36 & $57 \%$ & $\mathrm{HJU}$ & 58 & 23 & $40 \%$ & 3.7 & 0.055 & $M$ & $R>U$ \\
\hline & HMR & 51 & 16 & $31 \%$ & $\mathrm{HMU}$ & 70 & 19 & $27 \%$ & 0.3 & 0.61 & & \\
\hline ICC-A & MJR & 119 & 30 & $25 \%$ & MJU & 96 & $\mathrm{I}$ & $1 \%$ & 25.2 & 0 & & $R>U$ \\
\hline \multirow[t]{3}{*}{ ICC > = I } & MMR & 38 & 16 & $42 \%$ & MMU & 89 & 3 & $3 \%$ & 31.4 & 0 & & $R>U$ \\
\hline & $\mathrm{HJR}$ & 62 & 10 & $16 \%$ & $\mathrm{HJU}$ & 58 & 2 & $3 \%$ & 4.0 & 0.044 & & $R>U$ \\
\hline & HMR & 50 & 19 & $38 \%$ & $\mathrm{HMU}$ & 70 & 9 & $13 \%$ & 10.3 & 0.001 & & $R>U$ \\
\hline ICC-M & MJR & 119 & 82 & $69 \%$ & MJU & 96 & 51 & $53 \%$ & 5.6 & 0.018 & & $\mathrm{R}>\mathrm{U}$ \\
\hline$M=0.80-0.99$ & MMR & 38 & 22 & $58 \%$ & MMU & 89 & 76 & $85 \%$ & II.4 & 0.001 & & $U>R$ \\
\hline \multirow[t]{2}{*}{$\mathrm{H}=0.90-0.99$} & $\mathrm{HJR}$ & 62 & 45 & $73 \%$ & $\mathrm{HJU}$ & 58 & 27 & $47 \%$ & 8.5 & 0.004 & & $R>U$ \\
\hline & HMR & 50 & 29 & $58 \%$ & HMU & 70 & 46 & $66 \%$ & 0.7 & 0.39 & & \\
\hline Cint-A & MJR & 119 & 81 & $68 \%$ & MJU & 96 & 24 & $25 \%$ & 39.4 & 0 & & $R>U$ \\
\hline$M>88 \mathrm{~cm}$ & MMR & 38 & 30 & $79 \%$ & MMU & 89 & 57 & $64 \%$ & 2.7 & 0.098 & M & $R>U$ \\
\hline \multirow[t]{2}{*}{$\mathrm{H}>102 \mathrm{~cm}$} & $\mathrm{HJR}$ & 63 & 7 & $11 \%$ & $\mathrm{HJU}$ & 58 & 5 & $9 \%$ & 0.2 & 0.65 & & \\
\hline & HMR & 50 & 12 & $24 \%$ & HMU & 70 & 12 & $17 \%$ & 0.9 & 0.35 & & \\
\hline Cint-M & MJR & 119 & 22 & $18 \%$ & MJU & 96 & 32 & $33 \%$ & 6.2 & 0.013 & & $U>R$ \\
\hline$M=80-88 \mathrm{~cm}$ & MMR & 38 & 7 & $18 \%$ & $\mathrm{MMU}$ & 89 & 20 & $22 \%$ & 0.3 & 0.61 & & \\
\hline \multirow[t]{2}{*}{$\mathrm{H}=94-102 \mathrm{~cm}$} & $\mathrm{HJR}$ & 63 & 20 & $32 \%$ & $\mathrm{HJU}$ & 58 & 7 & $12 \%$ & 6.7 & 0.009 & & $\mathrm{R}>\mathrm{U}$ \\
\hline & HMR & 50 & 15 & $30 \%$ & HMU & 70 & 18 & $26 \%$ & 0.3 & 0.60 & & \\
\hline Hipertensión & MJR & 119 & 4 & $3 \%$ & MJU & 96 & 9 & $9 \%$ & 3.4 & 0.066 & $M$ & $U>R$ \\
\hline PAS=> I40 ó & MMR & 38 & 7 & $18 \%$ & MMU & 89 & 32 & $36 \%$ & 3.8 & 0.050 & $M$ & $U>R$ \\
\hline \multirow[t]{2}{*}{$\underline{P A D}=>90$} & $\mathrm{HJR}$ & 63 & 3 & $5 \%$ & $\mathrm{HJU}$ & 58 & 9 & $16 \%$ & 3.8 & 0.051 & $M$ & $U>R$ \\
\hline & HMR & 51 & 12 & $24 \%$ & HMU & 70 & 23 & $33 \%$ & 1.2 & 0.26 & & \\
\hline
\end{tabular}

$\mathrm{NI}=$ número de sujetos en el grupo; $\mathrm{N} 2$ = número de sujetos positivos a la anormalidad

Prevalencia $=\%$ de anormalidad ( 100 por $\mathrm{N} 2$ entre $\mathrm{NI})$

$\mathrm{Chi}=$ valor de la prueba de chi cuadrada de diferencias rural-urbana al interior de los grupos

Se añade una $M$ si la $p$ es marginal $(p=0.05$ a 0.10$)$

$M J R$ y $M J U=$ mujeres jóvenes rurales y urbanas; $M M R$ y $M M U$ = mujeres maduras rurales $y$ urbanas

$\mathrm{HJR}$ y $\mathrm{HJU}=$ hombres jóvenes rurales y urbanos; $\mathrm{HMR}$ y $\mathrm{HMU}=$ hombres maduros rurales $\mathrm{y}$ urbanos 
con valores inferiores a las urbanas en IMC-A e IMCM. No obstante, pese a estas discrepancias grupales, en general parece ser que tanto los de origen rural como los urbanos tienen prevalencias similares de obesidad y sobrepeso.

Las prevalencias de anormalidades de ICC-A fueron muy altas en rurales (16 a 42\%) en comparación con los urbanos (1 a 13\% ), de modo que todas las comparaciones rural-urbanas fueron significativamente diferentes. No ocurrió lo mismo con respecto a la prevalencia de anormalidades ICC-M, donde la diferencia Rur $>$ Urb sólo se mantuvo en los grupos de jóvenes; en los grupos maduros la tendencia fue contraria ( $\mathrm{Urb}>\mathrm{Rur}$ ). Es posible que esta inversión del sentido de la diferencia rural-urbana haya obedecido a la muy alta prevalencia de ICC-A en los grupos rurales maduros, lo cual dejó una proporción baja de casos con ICC-M anormal. Las frecuencias de anormalidad de cintura mostradas en el cuadro II van de acuerdo con lo observado en el cuadro I de que los altos promedios de ICC se deben, primordialmente, a mayor cintura de los grupos rurales. Empero, las diferencias en las frecuencias de anormalidad estuvieron menos claras, ya que sólo las mujeres jóvenes mostraron una relación Rur > Urb en Cint-A. En contraposición, en Cint-M hubo también diferencias significativas en mujeres y hombres jóvenes, pero en sentido opuesto (mujeres jóvenes con Rur $>$ Urb pero Urb $>$ Rur en hombres jóvenes). Por otra parte, este cambio en el sentido de la diferencia rural-urbana en Cint-M pareció deberse a un fenómeno similar al descrito anteriormente para ICC-M, esto es, las prevalencias muy altas de positividad de Cint-A en mujeres rurales drenaron los casos de riesgo medio en Cint-M. Todo lo anterior llevó a la conclusión de que la prevalencia de anormalidades de cintura y de ICC de los sujetos rurales fue, a todas luces, mayor que la de los urbanos.

Por su parte, la hipertensión osciló entre 3 y 16\% en los grupos jóvenes, y entre 18 y $36 \%$ en los maduros. Los grupos urbanos mostraron una tendencia a mayor prevalencia de hipertensión, ya que hubo una diferencia rural-urbana marginalmente significativa en tres de las cuatro comparaciones de hipertensión del cuadro II. La hipertensión alcanzó prevalencias de 9-16\% en jóvenes urbanos y de 36-33\% en maduros urbanos. Estos datos sugieren, por ende, que los urbanitas de Yucatán tienen una mayor prevalencia de hipertensión que los rurales, y que probablemente las diferencias grupales alcanzarían significancia si esta casuística fuera un poco mayor.

En síntesis, en el cuadro II se muestra que los rurales tuvieron prevalencias de anormalidades de IMC similares a los rurales, aunque tendieron a menos hipertensión sin que las diferencias alcanzaran significancia.
Fue notable la frecuencia alta de sobrepeso+obesidad en todos los grupos. Asimismo, hubo alta frecuencia de anormalidades de ICC y cintura en todos los grupos, especialmente en los rurales donde sobresalen las mujeres. Así entonces, prácticamente todos los sujetos rurales tuvieron problemas de cintura anormal, sobre todo las mujeres, aunque cabe destacar que los grupos urbanos no quedaron muy rezagados en sus frecuencias de anormalidades antropométricas. El patrón antropométrico, en consecuencia, se caracterizó en urbanos y rurales por sobrepeso y obesidad marcados, aunado a una adiposidad central alta que mostró diferencias según sexo y origen; por ejemplo, la cintura fue mayor en mujeres que en hombres, y mayor en rurales que en urbanitas.

\section{Discusión}

El presente trabajo debe ser visto como una primera aproximación, ya que los segmentos rural y urbano del estudio no son estrictamente comparables debido al muestreo (urbano polietápico vs. rural familiar), lo cual obedeció a que en el grupo rural se iban a incluir estudios de genética de población que no fue posible realizar por causas ajenas al control de los autores. En el estudio rural se terminó con una muestra que representa la tercera parte del universo poblacional. En opinión de los autores, este hecho compensa en parte el que la muestra rural no hubiera sido seleccionada en forma aleatoria. Por ello, se considera que los resultados en rurales son una primera aproximación aceptable a las prevalencias de obesidad e hipertensión en la población rural de Yucatán. Por otra parte, la comparatividad rural-urbana se mantuvo en lo que respecta a metodologías de las mediciones ya que, con excepción de la medición de la presión arterial (random-cero vs. digital), todas fueron realizadas previa estandarización. En el caso de la medición de la presión arterial se realizó un ajuste para hacer equivalentes los datos random-cero con Omron, empleando los aparatos usados en las encuestas.

\section{Obesidad y morfología corporal}

Tanto los sujetos rurales como los urbanos mostraron una morfología corporal caracterizada por baja talla y obesidad con adiposidad central elevada. Este patrón fue más acentuado en los grupos rurales y en las mujeres. El sobrepeso y la obesidad afectaron a más de la mitad de los sujetos, si bien las frecuencias de este estudio pudieran ser menores ya que se dice que el IMC sobreestima sobrepeso y obesidad en poblaciones de talla baja. ${ }^{12}$

En el cuadro III se compara la prevalencia de sobrepeso y obesidad de los ocho grupos de sexo- 
edad-origen con las cifras de las encuestas nacionales mexicanas en urbanos (Encuesta Nacional de Enfermedades Crónicas-ENEC 1993) y en urbanos y rurales (Encuesta Nacional de Salud-ENSA 2000). ${ }^{13,14}$ Para explorar diferencias atribuibles a etnicidad y a variaciones de ambiente y cultura, en el cuadro III se incluyeron resultados obtenidos por los autores, con la misma metodología, en comunidades rurales de la Mixteca Alta de Oaxaca. ${ }^{*}$ Los mixtecos fueron residentes de pequeñas comunidades dispersas asentadas en un altiplano a más de 2500 metros, lo cual implica el recorrido de distancias grandes y la realización de trabajo físico intenso. Estos datos de obesidad rural y urbana fueron mayores que los de los mixtecos en los ocho grupos, y mayores que los de la ENEC en seis grupos, pero no hubo prácticamente ninguna diferencia con respecto a la ENSA. En sobrepeso (excluida obesidad), los grupos de hombres mostraron tener más sobrepeso que los mixtecos, mas no así las mujeres. La unión de obesidad+sobrepeso mostró un patrón similar al de obesidad: los ocho grupos mayores que los mixtecos, mayores que la ENEC en seis grupos, e inclusive aparecieron algunas diferencias de mayor prevalencia que la ENSA. Se puede apreciar que Yucatán parece estar muy cercano a los datos de la encuesta ENSA y que sus prevalencias de obesidad (mas no de sobrepeso) superan a las de la ENEC.

En relación con la talla, se observaron diferencias promedio muy claras en función de la edad; así por ejemplo, entre los urbanos, los jóvenes superaron en 5.4 $\mathrm{cm}$ a los maduros, y en las mujeres urbanas hubo una diferencia de $3.3 \mathrm{~cm}$ entre jóvenes y maduras (cuadro I). Las diferencias de talla en los grupos rurales fueron menores. Esto habla de una posible aceleración en los cambios de talla en el medio urbano de Yucatán y, en menor grado, en el medio rural. La aceleración del crecimiento de talla en el ambiente urbano es un fenómeno que se ha dado en otras regiones del país. ${ }^{\ddagger}$ Por otra parte, la literatura menciona factores positivos para el ambiente urbano, entre ellos, mejor salud y nutrición prenatales y postnatales, mejor calidad de proteínas de la dieta y, en general, mejor saneamiento con la consiguiente disminución de la morbilidad infecciosa. ${ }^{15} \mathrm{Sin}$ importar cuáles hayan sido los procesos participantes

\footnotetext{
* Fernández V, Loría A, Pardío J, Arroyo P. Prevalencia de hipertensión en población adulta de la Mixteca Alta de Oaxaca. Documento no publicado, 2005.

Fernández V, Loría A, Pardío J, Arroyo P. Prevalencia de sobrepeso y obesidad en población adulta de la Mixteca Alta de Oaxaca. Documento no publicado, 2005.

₹ Arroyo P, Fernández V. Diferencia intergeneracional de talla en una muestra de la población adulta de México. Documento no publicado, 2005.
}

en las diferencias urbanas de talla joven-maduro en Yucatán, es probable que hayan actuado al margen de los factores que dan lugar a la obesidad ya que ésta, contrariamente a las diferencias de talla, afectó más fuertemente al sector rural que al urbano (IMC significativamente mayor y talla significativamente menor en jóvenes rurales que en sus contrapartes urbanas) (cuadro I). Si se asume que la talla de la población adulta rural es un indicador del estado de nutrición deficiente previo, es claro que al haber estado esta población expuesta a una disponibilidad de energía más amplia y a cambios en sus patrones de actividad física, se expresen de manera más acentuada los rasgos antropométricos característicos del síndrome metabólico (IMC y cintura anormales).

En el cuadro IV se comparan los datos de este estudio correspondientes a circunferencia de cintura, con los notificados en la Encuesta Nacional de Salud y Nutrición 2006-ENSANUT $2006{ }^{16}$ y con el grupo de mixtecos. Los hombres, tanto rurales como urbanos, presentaron una prevalencia de cintura $>102 \mathrm{~cm}$ que es significativamente menor que la indicada en la ENSANUT, pero mayor que la de mixtecos. Si el corte se hace con cintura mayor de $90 \mathrm{~cm}$, como es el caso de la ENSANUT, ya no hay diferencias con los datos de esta última encuesta, pero siguen siendo significativamente mayores que en los mixtecos. En las mujeres, las anormalidades de cintura mostraron patrones diferentes a los de los hombres: las rurales jóvenes tuvieron prevalencias mayores que las notificadas en la ENSANUT y que las de mixtecos, en tanto que las urbanas jóvenes sólo superaron a las mixtecas.

\section{Presión arterial}

A este respecto, fue claro que las cifras promedio de PAS fueron más elevadas en los sujetos urbanos que en los rurales, al igual que la prevalencia de hipertensión arterial. Estas observaciones son consistentes con la tesis de un efecto favorecedor de que el ascenso de la presión arterial con la edad es más intenso en los residentes urbanos que en los rurales. ${ }^{17,18}$ Por otra parte, las diferencias rural-urbanas de PAD no fueron significativas, lo cual sugiere que las diferencias ruralurbanas de prevalencia de hipertensión están determinadas, sobre todo, por la presión sistólica. En lo que concierne al efecto de la edad sobre la presión arterial, los resultados encontrados son consistentes con los de otros autores como Whelton, ${ }^{18}$ quien enfatiza esta influencia de edad sobre PAS, de modo que es mayor en la vejez que en edad adulta.

Pollard y colaboradores, ${ }^{19}$ en un estudio sobre la distribución geográfica de la variabilidad de la presión 


\section{Cuadro IV}

Comparación de ANORMALIdAdES de CIRCUNFERENCIA DE CINTURA (CC) VERSUS UNA ENCUESTA NACIONAL (ENSA) Y UNA EN INDÍGENAS DE OAXACA

\begin{tabular}{|c|c|c|c|c|c|}
\hline & Población & Edad & $N$ & $\%$ & $\%$ \\
\hline Mujeres & & & & $C C>88$ & $C C>80$ \\
\hline Presente estudio & Rural & $20-49$ & 119 & $68.1^{\mathrm{ac}}$ & $86.6^{\mathrm{a} * \mathrm{c}}$ \\
\hline Presente estudio & Urbana & $20-49$ & 96 & $25.0 \%$ & $58.3^{c}$ \\
\hline Mixtecos, Oaxaca ${ }^{\ddagger}$ & Rural & $20-49$ & 124 & 14.5 & 39.5 \\
\hline ENSANUT ${ }^{16}$ & Ambas & $20-49$ & 13,787 & 57.5 & 77.7 \\
\hline Presente estudio & Rural & $50-75$ & 38 & $78.9^{c}$ & $97.4^{c}$ \\
\hline Presente estudio & Urbana & $50-75$ & 89 & $64.0^{\mathrm{bc}}$ & $86.5^{b c}$ \\
\hline Mixtecos, Oaxaca ${ }^{\ddagger}$ & Rural & $50-75$ & 45 & 24.4 & 51.1 \\
\hline ENSANUT $^{16}$ & Ambas & $50-79$ & 5,276 & 81.4 & 92.2 \\
\hline Hombres & & & & $C C>102$ & $C C>90$ \\
\hline Presente estudio & Rural & $20-49$ & 63 & $11.1^{b c}$ & $63.5^{c}$ \\
\hline Presente estudio & Urbana & $20-49$ & 58 & $8.6^{\mathrm{bc} *}$ & $44.8^{c}$ \\
\hline Mixtecos, Oaxaca ${ }^{\ddagger}$ & Rural & $20-49$ & 66 & 1.5 & 12.1 \\
\hline ENSANUT $^{16}$ & Ambas & $20-49$ & 8,361 & 20.8 & 60.4 \\
\hline Presente estudio & Rural & $50-75$ & 50 & $24.0^{\mathrm{bc}}$ & $56.0^{c}$ \\
\hline Presente estudio & Urbana & $50-75$ & 70 & $17 .\left.\right|^{\mathrm{bc}}$ & $51.4^{\mathrm{c}}$ \\
\hline Mixtecos, Oaxaca ${ }^{\ddagger}$ & Rural & $50-75$ & 66 & 0.0 & 0.0 \\
\hline ENSANUT $^{16}$ & Ambas & $50-79$ & 3,919 & 31.4 & 72.3 \\
\hline
\end{tabular}

Los resultados presentados aquí sugieren que en la población urbana y rural de Yucatán la obesidad es un problema de salud de primera importancia, ya que las complicaciones de comorbilidades, como la aterosclerosis y la diabetes, se ubican desde hace una década en los primeros lugares de la patología regional..$^{22}$ Existen indicios de que la obesidad, como fenómeno epidémico en Yucatán, es relativamente reciente. Por ejemplo, apenas en 1963 Chávez y colaboradores ${ }^{23}$ encontraron obesidad en sólo $6 \%$ de las mujeres adultas de comunidades rurales mayas localizadas en la misma zona de la península donde se ubican las comunidades motivo de este trabajo. Dichos autores no hallaron obesidad en hombres y el patrón epidemiológico dominante fue el de desnutrición asociada con deficiencia de niacina. Estudios posteriores realizados en la década de 1990 muestran, contrariamente, una frecuencia alta de obesidad en población yucateca asistente a instituciones hospitalarias. ${ }^{24}$

Un cambio de la morfología corporal de la población de Yucatán, como lo es un incremento de talla y una menor circunferencia abdominal, sólo puede explicarse por una modificación importante en las condiciones materiales que transformaron el estilo de vida de toda la población. En la península de Yucatán, el único cambio reciente de tal magnitud es la desaparición del cultivo del henequén como actividad económica primaria ${ }^{25}$ fenómeno que determinó la migración masiva del campo a la ciudad. Este cambio se dio en un lapso breve, en una región de México que incluye a Yucatán y que presenta rasgos físicos que la diferencian de otras regiones. El estado de Yucatán tenía 1556622 habitantes en 2001 y estaba conformado por 106 municipios, 11 de los cuales concentraban $65.9 \%$ de la población estatal. ${ }^{26}$ La comunicación con el centro de México se hizo por mar hasta la década de los años cuarenta del siglo XX cuando se construyó el ferrocarril, y se intensificó en los años cincuenta con la apertura de la carretera que une a la entidad con el resto del país. ${ }^{24}$ Esta apertura coincidió con el crecimiento de la población urbana de Mérida, que se aceleró con la caída del cultivo del henequén que, hasta hace 25 años, seguía siendo el producto agrícola yucateco de mayor valor económico.

En la década de 1950, la densidad de población de Yucatán era semejante a la del país: 13.2 vs 13.5 ha- 
CuadroV

Comparación de PREVALENCIA de hiPERTENSIÓn VERSUS UNA ENCUESTA NACIONAL (ENEC) Y UNA EN INDÍGENAS DE OAXACA

\begin{tabular}{|c|c|c|c|c|}
\hline \multicolumn{4}{|l|}{ Mujeres } & $\%$ \\
\hline Presente estudio & Rural & $20-49$ & 119 & $3.4^{\mathrm{ab}}$ \\
\hline Presente estudio & Urbana & $20-49$ & 96 & $9.4^{\mathrm{ab}}$ \\
\hline Mixtecos, Oaxaca ${ }^{\ddagger}$ & Rural & $20-49$ & 124 & 1.6 \\
\hline ENEC $^{21}$ & Urbana & $20-49$ & 6,837 & 20.4 \\
\hline Presente estudio & Rural & $50-75$ & 38 & $18.4^{\mathrm{ab} *}$ \\
\hline Presente estudio & Urbana & $50-75$ & 89 & $36.0^{\mathrm{ab}}$ \\
\hline Mixtecos, Oaxaca ${ }^{\ddagger}$ & Rural & $50-75$ & 45 & 6.7 \\
\hline ENEC $^{21}$ & Urbana & $50-69$ & 1,767 & 57.9 \\
\hline \multicolumn{5}{|l|}{ Hombres } \\
\hline Presente estudio & Rural & $20-49$ & 63 & $4.8^{a}$ \\
\hline Presente estudio & Urbana & $20-49$ & 58 & $15.5^{\mathrm{ab}}$ \\
\hline Mixtecos, Oaxaca ${ }^{\ddagger}$ & Rural & $20-49$ & 66 & 1.5 \\
\hline ENEC $^{21}$ & Urbana & $20-49$ & 4,808 & 32.2 \\
\hline Presente estudio & Rural & $50-75$ & 51 & $23.5^{\mathrm{a}}$ \\
\hline Presente estudio & Urbana & $50-75$ & 70 & $32.9^{\mathrm{ab}}$ \\
\hline Mixtecos, Oaxaca ${ }^{\ddagger}$ & Rural & $50-75$ & 32 & 9.4 \\
\hline ENEC $^{21}$ & Urbana & $50-69$ & 1,245 & 58.2 \\
\hline \multicolumn{5}{|c|}{$\begin{array}{l}\text { * Se añade un asterisco a la letra si la diferencia fue marginal } \\
(\mathrm{P}=0.05-0.10)\end{array}$} \\
\hline \multicolumn{5}{|c|}{$\begin{array}{l}\text { ‡ Fernández V y cols. Prevalencia de sobrepeso y obesidad en población } \\
\text { adulta de la Mixteca Alta de Oaxaca. Datos no publicados. } 2005\end{array}$} \\
\hline \multicolumn{5}{|c|}{$\begin{aligned} \mathrm{a}= & \text { significativamente MENOR que ENEC } \\
& \text { (prueba de diferencia de proporciones) }\end{aligned}$} \\
\hline$b=$ significativamente & AYYR que & xtecos & a chi & \\
\hline
\end{tabular}

bitantes por $\mathrm{km}^{2}$. No obstante, en los años ochenta, la densidad de Yucatán se incrementó a 34.1, superando la del resto del país (27.7). En 1990, la fracción urbana nacional constituía 71\% de la población; en el caso de Yucatán, correspondía a 79\%, de la cual 52\% residía en la ciudad de Mérida. ${ }^{27}$ Diez años después, en 2000, las cifras respectivas fueron de 75, 81 y $52 \%$. La desaparición del cultivo del henequén fue un proceso relativamente rápido que se aceleró a partir de los años sesenta debido a su pérdida de competitividad frente a otros productores y al advenimiento de las fibras sintéticas. En un periodo de transición que tuvo lugar en la década de los años setenta, el impacto de esta transición se amortiguó con un fuerte subsidio económico que mantuvo la productividad agrícola hasta cierto nivel. Sin embargo, ese equilibrio terminó por romperse con el cambio de política económica del Estado mexicano en los años ochenta, que aceleró el desplazamiento de la población del campo a Mérida y a otras regiones de México y, más recientemente, a Estados Unidos de América. El subsidio económico a las comunidades rurales se transfirió a las familias insertadas en la economía urbana de Mérida, la cual sufrió una expansión comercial y turística que atrajo migrantes rurales.

Es probable que la elevada prevalencia de obesidad en Yucatán esté relacionada con esa ruptura del estilo de vida, caracterizada por la disminución del tiempo dedicado a actividades agrícolas intensas y prolongadas, y acoplada con una mejoría de la economía rural que facilitó el acceso a una dieta más diversa y abundante. Se sugiere que estos mismos factores han operado en la población urbana, y que en ambos ambientes han coadyuvado otros factores como el consumo de una dieta de alta densidad energética característica de la cultura culinaria de Yucatán y un clima que limita las actividades físicas de sus pobladores.

En cuanto a hipertensión, las principales conclusiones de este análisis son: a) la prevalencia de hipertensión en Yucatán fue más baja que las cifras correspondientes para todo el país; b) la prevalencia de hipertensión en los grupos rurales fue baja, aunque mayor que la encontrada en mixtecos rurales; c) hubo prevalencia relativamente baja de hipertensión en Yucatán, pese a que se ha observado la presencia de varios factores de riesgo de hipertensión, entre ellos, elevada prevalencia de obesidad de tipo central, sobrepeso elevado, consumo de una dieta de alta densidad energética y patrones sedentarios de actividad física, especialmente en las mujeres. El consumo de sal, como factor de riesgo de hipertensión en esta población, no se investigó aquí. La posibilidad de que las poblaciones maya y mestiza de Yucatán presenten menos riesgo de hipertensión arterial, aun en presencia de factores adversos, requiere confirmación.

\section{Agradecimientos}

Esta investigación se llevó a cabo gracias al financiamiento aportado por el Fondo Nestlé para la Nutrición, de la Fundación Mexicana para la Salud.

\section{Referencias}

I. Grundy SM. Multifactorial causation of obesity: implications for prevention. Am J Clin Nutr 1998;67 suppl 3:S563-S572.

2. Reaven $\mathrm{G}$. Role of insulin resistance in human disease. Diabetes 1988;37:1595-1607.

3. De Caterina R, Zampolli A, Del Turco S, Madonna R, Massaro M. Nutritional mechanisms that influence cardiovascular disease.Am J Clin Nutr 2006;83 suppl:S42I-S426.

4. Drewnowski A, Popkin BM. The nutrition transition: new trends in the global diet. Nutr Rev 1997;55:3I-43.

5. INEGI. XI Censo General de Población y Vivienda. México: INEGI, 1992. 
6. López HR.AMAl: distribución de niveles socioeconómicos en el México urbano. Datos, diagnósticos, tendencias 2006;13:1-7.

7.World Health Organization. Obesity: preventing and managing the global epidemic. Geneva:WHO, 1998.

8. World Health Organization. Physical status: the use and interpretation of anthropometry. Report of a WHO Expert Committee. Geneva:WHO, 1995. 9. The sixth report of the Joint National Committee on the Detection, Evaluation, and Treatment of High Blood Pressure. National Institutes of Health, National Heart, Lung and Blood Institute. NIH publication No. 98-4080, 1997.

10. Fernández-García V, Zárate-Grajales RA. Estudio comparativo de tres tipos de esfigmomanómetros, en sujetos con diferentes cifras de presión arterial. Enfermería universitaria 2006;3:8-13.

I I. Fleiss JL. An introduction to applied probability. Statistical methods for rates and proportions. 2a. edición. New York:JWiley, 1981:13-14.

12. Long AE, Prewitt JS, Kaufman JS, Rotimi CN, Cooper R, McGee DL.

Weight-height relationships among eight populations of West African origin: the case against constant BMI standards. Int J Obes 1998;22:842-846. 13.Arroyo P, Loría A, Fernández V, Flegal K, Kuri-Morales P, Olaiz G et al. Prevalence of pre-obesity and obesity in urban adult Mexicans in comparison with other large surveys. Obes Res 2000;8: 179-185.

14. Olaiz G, Rojas R, Barquera S, Shamah T,Aguilar C, Cravioto P. Encuesta Nacional de Salud 2000. La salud de los adultos, Vol. 2. Cuernavaca: Instituto Nacional de Salud Pública, 2003.

15. Eveleth PB, Tanner JM. Environmental influence on growth. Worldwide variation in human growth. 2a. edición. Cambridge: Cambridge University Press, 1990;219-223.

16. Olaiz-Fernández G, Rivera-Dommarco J, Shamah-Levy T, Rojas R, Villalpando-Hernández S, Hernández-Avila M, et al. Encuesta Nacional de Salud y Nutrición 2006. Cuernavaca, México: Instituto Nacional de Salud Pública, 2006.
17. Dressler W. Modernization, stress, and blood pressure: new directions in research. Human Biol 1999;71:583-605.

18. Whelton PK. Epidemiology of hypertension. Lancet 1994;344:10I-106. 19. Pollard TM, Brush G, Harrison GA. Geographic distributions of withinpopulation variability in blood pressure. Human Biol 1991;63:643-66I.

20. Salmond CE, Joseph JG, Prior IA, Stanley DG, Wessen AF. Longitudinal analysis of the relationship between blood pressure and migration:Tokelau Island Migrant Study.Am J Epidemiol 1985; I22:291-30I.

21.Arroyo P, Fernández V, Loría A, Kuri-Morales P, Orozco-Rivadeneyra S,

Olaiz $G$ et al. Hypertension in urban Mexico: the 1992-93 national survey of chronic diseases.J Hum Hypertens 1999;13:67|-675.

22. Secretaría de Salud. Dirección General de Estadística e Informática.A. Mortalidad 1999. México: SSA, 2001.

23. Chávez A, Balam G, Zubirán S. Estudios epidemiológicos de la diabetes en tres comunidades de la zona henequenera del estado de Yucatán. Rev Invest Clin 1963;15:333-344.

24.Vargas-Ancona L. Epidemiología de la diabetes mellitus, intolerancia a la glucosa y factores de riesgo aterogénico en Yucatán, México. Rev Biomédica 1994:5:151-159.

25. Escalante R. The State and henequen production in Yucatan 19551980. Occasional Papers. London: University of London. Institute of Latin American Studies, 1988.

26. Instituto Nacional de Estadística, Geografía e Informática. Perspectiva estadística de Yucatán. México: INEGI, 1997.

27. Instituto Nacional de Estadística, Geografía e Informática. Anuario Estadístico de los Estados Unidos Mexicanos. México: INEGI, 2000. 